\title{
Continuous Time Random Walks with Reactions Forcing and Trapping
}

\author{
C. N. Angstmann, I. C. Donnelly, B. I. Henry * \\ School of Mathematics and Statistics, University of New South Wales, Sydney, Australia
}

\begin{abstract}
One of the central results in Einstein's theory of Brownian motion is that the mean square displacement of a randomly moving Brownian particle scales linearly with time. Over the past few decades sophisticated experiments and data collection in numerous biological, physical and financial systems have revealed anomalous sub-diffusion in which the mean square displacement grows slower than linearly with time. A major theoretical challenge has been to derive the appropriate evolution equation for the probability density function of sub-diffusion taking into account further complications from force fields and reactions. Here we present a derivation of the generalised master equation for an ensemble of particles undergoing reactions whilst being subject to an external force field. From this general equation we show reductions to a range of well known special cases, including the fractional reaction diffusion equation and the fractional Fokker-Planck equation.
\end{abstract}

Keywords and phrases: fractional diffusion, reaction-diffusion, random walk, FokkerPlanck equation, stochastic process

Mathematics Subject Classification: 60G22, 35K57, 35Q84, 82C41, 35R11, 60J60

\section{Introduction}

The Fokker-Planck equation, or forward Kolmogorov equation, has long been used to model the evolution of the probability density of a particle that is undergoing Brownian motion (standard diffusion) subject to an external force [1-3]. The fractional Fokker-Planck equation for a particle undergoing sub-diffusion in a spatially varying force field was first derived from the spatial continuum limit of a biased continuous time random walk (CTRW) in [4]. The corresponding equation for sub-diffusion in an external time-dependent force field was derived in [5] and the equivalence to a subordinated Langevin equation was shown in [6]. The fractional Fokker-Planck equation with both time- and space-dependent forces was derived in [7]. Other fractional Fokker-Planck equations have been derived outside of a CTRW framework [8-10].

CTRWs have also been used to generalise reaction-diffusion models to systems with anomalous diffusion. Master equations that govern the time evolution of the density of particles have been derived for a range of cases. This was first considered in [11] with mass action reaction terms added to the sub-diffusion term. A sophisticated treatment properly incorporating linear reaction kinetics in the CTRW framework

*Corresponding author. E-mail: b.henry@unsw.edu.au 
was derived in [12-14]. This was extended to non-linear reactions in [15]. An alternative derivation with pure death processes was given earlier in [16].

The CTRW model for sub-diffusion exhibits non-ergodic behaviour. The ensemble average mean square displacement scales as a sub-linear power law in time whereas time average mean square displacements scale approximately linearly with time, with different diffusion coefficients for different realisations $[17,18]$. An alternate model for sub-diffusion is fractional Brownian motion (fBm). This model has a Gaussian probability density function with a time dependent diffusion coefficient and it exhibits ergodic behaviour [19]. In this article we have confined our attention to the CTRW model, however tests to distinguish between different models for sub-diffusive behaviour are an active area of research $[18,20]$.

Among the experimental systems that exhibit diffusion, reactions and forcing, the motility of populations of cells is especially interesting because of the confounding aspect of chemotaxis, where particles experience a force in proportion to a chemical gradient. The governing equations to model sub-diffusion with linear reactions and chemotaxis have been derived in [21]. Sub-diffusion with non-linear reactions and chemotaxis was considered in [22].

Fractional Fokker-Planck equations and fractional reaction-diffusion equations have become increasingly important because of the growing recognition that anomalous diffusion is ubiquitous [23]. Anomalous sub-diffusion is widespread in biological systems due to traps and crowding effects. In such systems the traps may be structural traps $[24,30]$ or binding spots [26]. Anomalous sub-diffusion has been measured in protein movements in cells [27], in movement of lipid granules in yeast cells [28] and movements of molecules in spiny neuronal dendrites $[29,30]$. The consideration of the possible anomalous diffusion of ions in nerve cells prompted the development of a fractional cable equation to model the electrical properties of neurons [31-33]. The anomalous diffusion of molecules in cell membranes has led to a re-consideration of recovery curves obtained from fluorescence recovery after photobleaching (FRAP) experiments [34].

As observations of anomalous diffusion are reported in a wider variety of systems, the master equations and Fokker-Planck equations that model the time evolution of these systems need to be more general to accommodate the observations. Here we present the full derivation of the generalized master equations and associated fractional Fokker-Planck equations, starting from the underlying stochastic process of a CTRW in space- and time-dependent force fields with reactions. We show reductions to the fractional reaction-diffusion equation and the fractional Fokker-Planck equation as special cases.

\section{Generalized Master Equation Derivation}

\subsection{Reaction Kinetics and Forcing}

In reaction-diffusion processes, reaction kinetics are usually incorporated as an additive term through the law of mass action [35-37]. However, in reaction sub-diffusion processes the reaction kinetics and the transport terms become entwined $[12,13,15]$. In the following we separate the reaction kinetics as birth and death processes $[15,38]$. Death may be due to the particle changing type in which case there will be a corresponding birth. We assume that the probability of a particle dying between $t$ and $t+\delta t$ is given by $\omega(x, t) \delta t+o(\delta t)$. It follows that the probability of the particle surviving to time $t$ is

$$
\theta(x, t, s)=e^{-\int_{s}^{t} \omega\left(x, t^{\prime}\right) d t^{\prime}}
$$

and time till death is exponentially distributed with a non-uniform rate parameter. We define a probability of a particle being created between times $t$ and $t+\delta t$ as $\eta(x, t) \delta t+o(\delta t)$.

As the simplest example, consider the reaction $A \rightarrow B$. In this reaction we have death of particle $A$ with a corresponding birth of particle $B$. From the law of mass action, the time evolution of concentrations of particles of type $A$ and $B$ are $\frac{d A}{d t}=-k A$ and $\frac{d B}{d t}=k A$ respectively. The probability of a particle of type $A$ dying between $t$ and $t+\delta t$ in this case is the per capita death rate $k$ times the time interval $\delta t$; thus $\omega(x, t)=k$. The probability of a particle of type $B$ being born between times $t$ and $t+\delta t$ is equal to per capita death rate $k$ times the concentration of $A$; thus $\eta(x, t)=k A$. 
The effect of a force field on the particle can be incorporated into the jump density $[4,7]$. The force field biases the direction that the particle jumps as a function of both $x$ and $t$.

\subsection{Single Particle Master Equation}

We start by deriving the master equation for a single particle undergoing a CTRW with a pure death process. This derivation follows the derivation presented in [39] with the added complications from the space- and time-dependent force field. As we want to start from the underlying stochastic process we consider the arriving flux density of the particle at point $x$ at time $t$ after it has taken no jumps given that the particle was initially at point $x_{0}$ at time 0

$$
q_{0}\left(x, t \mid x_{0}, 0\right)=\delta_{x, x_{0}} \delta\left(t-0^{+}\right)
$$

In general the flux after $n+1$ steps is given by recursion equation

$$
q_{n+1}\left(x, t \mid x_{0}, 0\right)=\sum_{x^{\prime}} \int_{0}^{t} \Psi\left(x, x^{\prime}, t, t^{\prime}\right) \theta\left(x^{\prime}, t, t^{\prime}\right) q_{n}\left(x^{\prime}, t^{\prime} \mid x_{0}, 0\right) d t^{\prime},
$$

where $\Psi\left(x, x^{\prime}, t, t^{\prime}\right)$ is the probability that a particle at $x^{\prime}$ at time $t^{\prime}$ jumps to $x$ at time $t$. We make the assumption that this may be expressed as a product of two independent densities; a jump density $\lambda\left(x, x^{\prime}, t\right)$ and a waiting time density $\psi\left(x^{\prime}, t-t^{\prime}\right)$ so that

$$
\Psi\left(x, x^{\prime}, t, t^{\prime}\right)=\lambda\left(x, x^{\prime}, t\right) \psi\left(x^{\prime}, t-t^{\prime}\right)
$$

where $\lambda$ and $\psi$ must satisfy the normalizations;

$$
\sum_{x} \lambda\left(x, x^{\prime}, t\right)=1 \quad \text { for fixed } x^{\prime} \text { and } t
$$

and

$$
\int_{t^{\prime}}^{\infty} \psi\left(x^{\prime}, t-t^{\prime}\right) d t=1 \quad \text { for fixed } x^{\prime} \text { and } t^{\prime} .
$$

The $x$ dependence in the waiting time density in Eq. (2.2) allows for spatially non-uniform trapping [40]. The forcing will be included through a bias in the jump density.

Following Scher and Lax [41], we sum over the number of jumps to derive the recurrence relation for the flux density of a particle arriving at point $x$ at time $t$ independent of the number of jumps taken

$$
q\left(x, t \mid x_{0}, 0\right)=\delta_{x, x_{0}} \delta\left(t-0^{+}\right)+\sum_{x^{\prime}} \int_{0}^{t} \Psi\left(x, x^{\prime}, t, t^{\prime}\right) \theta\left(x^{\prime}, t^{\prime}, t\right) q\left(x^{\prime}, t^{\prime} \mid x_{0}, 0\right) d t^{\prime}
$$

The probability of a particle being at point $x$ at time $t$ is given by

$$
\rho\left(x, t \mid x_{0}, 0\right)=\int_{0}^{t} \Phi\left(x, t-t^{\prime}\right) \theta\left(x, t, t^{\prime}\right) q\left(x, t^{\prime} \mid x_{0}, 0\right) d t^{\prime}
$$

where

$$
\Phi\left(x, t-t^{\prime}\right)=1-\int_{0}^{t-t^{\prime}} \psi\left(x, t^{\prime \prime}\right) d t^{\prime \prime}
$$

is the survival probability.

Care has to be taken due to the discontinuity at the boundary in the flux density, Eq. (2.3). Following Appendix we define

$$
q\left(x, t \mid x_{0}, 0\right)=\delta_{x, x_{0}} \delta\left(t-0^{+}\right)+q^{+}\left(x, t \mid x_{0}, 0\right)
$$


so that $q^{+}\left(x, t \mid x_{0}, 0\right)$ is right continuous at the $t=0$ boundary. Substituting this into Eq. (2.4) gives

$$
\rho\left(x, t \mid x_{0}, 0\right)=\delta_{x, x_{0}} \Phi(x, t) \theta(x, t, 0)+\int_{0}^{t} q^{+}\left(x, t^{\prime} \mid x_{0}, 0\right) \theta\left(x, t, t^{\prime}\right) \Phi\left(x, t-t^{\prime}\right) d t^{\prime} .
$$

Differentiating this equation with respect to time then gives

$$
\begin{aligned}
\frac{\partial \rho\left(x, t \mid, x_{0}, 0\right)}{\partial t}= & q^{+}\left(x, t \mid x_{0}, 0\right)-\int_{0}^{t} q\left(x, t^{\prime} \mid x_{0}, 0\right) \theta\left(x, t, t^{\prime}\right) \psi\left(x, t-t^{\prime}\right) d t^{\prime} \\
& -\omega(x, t) \rho\left(x, t \mid x_{0}, 0\right)-\delta_{x, x_{0}} \theta(x, t, 0) \psi(x, t)
\end{aligned}
$$

It is then possible to define the flux density leaving the point $x$ at time $t$ as

$$
\begin{aligned}
i\left(x, t \mid x_{0}, 0\right) & =\delta_{x, x_{0}} \theta(x, t, 0) \psi(x, t)+\int_{0}^{t} q^{+}\left(x, t^{\prime} \mid x_{0}, 0\right) \theta\left(x, t, t^{\prime}\right) \psi\left(x, t-t^{\prime}\right) d t^{\prime} \\
& =\int_{0}^{t} q\left(x, t^{\prime} \mid x_{0}, 0\right) \theta\left(x, t, t^{\prime}\right) \psi\left(x, t-t^{\prime}\right) d t^{\prime}
\end{aligned}
$$

This allow us to express the derivative of the density, Eq. (2.6), as a differences of fluxes

$$
\frac{\partial \rho\left(x, t \mid x_{0}, 0\right)}{\partial t}=q^{+}\left(x, t \mid x_{0}, 0\right)-i\left(x, t \mid x_{0}, 0\right)-\omega(x, t) \rho\left(x, t \mid x_{0}, 0\right)
$$

Given that the flux density for arriving at point $x$ at time $t$ can be expressed as the instantaneous flux that have left other points $x^{\prime}$ we have

$$
\frac{\partial \rho\left(x, t \mid x_{0}, 0\right)}{\partial t}=\sum_{x^{\prime}} \lambda\left(x, x^{\prime}, t\right) i\left(x^{\prime}, t \mid x_{0}, 0\right)-i\left(x, t \mid x_{0}, 0\right)-\omega(x, t) \rho\left(x, t \mid x_{0}, 0\right) .
$$

To express $i\left(x, t \mid x_{0}, 0\right)$ in terms of $\rho\left(x, t \mid x_{0}, 0\right)$ we use Laplace transform methods. We first note that due to the exponential form of Eq. (2.1)

$$
\theta(x, t, 0)=\theta\left(x, t^{\prime}, 0\right) \theta\left(x, t, t^{\prime}\right)
$$

It follows that we can rewrite Eq. (2.4) as

$$
\frac{\rho\left(x, t \mid x_{0}, 0\right)}{\theta(x, t, 0)}=\int_{0}^{t} \frac{q\left(x, t^{\prime} \mid x_{0}, 0\right)}{\theta\left(x, t^{\prime}, 0\right)} \Phi\left(x, t-t^{\prime}\right) d t^{\prime}
$$

and then take the Laplace transform to give

$$
\mathcal{L}\left\{\frac{\rho\left(x, t \mid x_{0}, 0\right)}{\theta(x, t, 0)}\right\}=\mathcal{L}\left\{\frac{q\left(x, t \mid x_{0}, 0\right)}{\theta(x, t, 0)}\right\} \hat{\Phi}(x, s)
$$

where $\mathcal{L}$ denotes the Laplace transform operator, the hat denotes a Laplace transformed function, and $s$ is the Laplace variable. Similarly we can rewrite Eq. (2.7) as

$$
\frac{i\left(x, t \mid x_{0}, 0\right)}{\theta(x, t, 0)}=\int_{0}^{t} \frac{q\left(x, t^{\prime} \mid x_{0}, 0\right)}{\theta\left(x, t^{\prime}, 0\right)} \psi\left(x, t-t^{\prime}\right) d t^{\prime}
$$

with Laplace transform

$$
\mathcal{L}\left\{\frac{i\left(x, t \mid x_{0}, 0\right)}{\theta(x, t, 0)}\right\}=\mathcal{L}\left\{\frac{q\left(x, t \mid x_{0}, 0\right)}{\theta(x, t, 0)}\right\} \hat{\psi}(x, s)
$$


Combining the two transformed equations, Eq.(2.10) and Eq.(2.11), and then inverting the transform gives

$$
i\left(x, t \mid x_{0}, 0\right)=\int_{0}^{t} K\left(x, t-t^{\prime}\right) \rho\left(x, t^{\prime} \mid x_{0}, 0\right) \theta\left(x, t, t^{\prime}\right) d t^{\prime},
$$

where $K(x, t)$ is defined by its Laplace transform

$$
\hat{K}(x, s)=\frac{\hat{\psi}(x, s)}{\hat{\Phi}(x, s)} .
$$

It is now possible to write Eq. (2.8) as the generalised master equation for a single particle with a probability of dying;

$$
\begin{aligned}
\frac{\partial \rho\left(x, t \mid, x_{0}, 0\right)}{\partial t}= & \sum_{x^{\prime}} \lambda\left(x, x^{\prime}, t\right) \int_{0}^{t} K\left(x^{\prime}, t-t^{\prime}\right) \rho\left(x^{\prime}, t^{\prime} \mid x_{0}, 0\right) \theta\left(x^{\prime}, t, t^{\prime}\right) d t^{\prime} \\
& -\int_{0}^{t} K\left(x, t-t^{\prime}\right) \rho\left(x, t^{\prime} \mid x_{0}, 0\right) \theta\left(x, t, t^{\prime}\right) d t^{\prime}-\omega(x, t) \rho\left(x, t \mid x_{0}, 0\right) .
\end{aligned}
$$

In the case of power law waiting times with nearest neighbour jumps in one dimension, Eq. (2.12) reduces to the mesoscopic fractional sub-diffusion equation with death processes, Eq. (29) in [16].

\subsection{Ensemble Master Equation}

The single particle master equation, Eq. (2.12), can be used to derive the generalised master equation for an ensemble of particles that are created and destroyed by a reaction process whilst being subjected to an external force field. We make the simplifying assumption that the newly created particles will be independent of any parent particles, i.e. they will draw a new waiting time. This is equivalent to Model $\mathrm{B}$ [15]. We define a probability of a particle being created between times $t$ and $t+\delta t$ as $\eta(x, t) \delta t+o(\delta t)$. It is then possible to write the density of particles at a point $x$ at time $t$ as

$$
u(x, t)=\sum_{x_{0}} \int_{0}^{t} \rho\left(x, t \mid x_{0}, t_{0}\right) \eta\left(x_{0}, t_{0}\right) d t_{0} .
$$

We can then differentiate this with respect to time and use the single particle master equation, Eq. (2.12) to arrive at

$$
\begin{aligned}
\frac{\partial u(x, t)}{\partial t}= & \sum_{x_{0}} \int_{0}^{t} \eta\left(x_{0}, t_{0}\right)\left[\sum_{x^{\prime}} \lambda\left(x, x^{\prime}, t\right) \int_{0}^{t} K\left(x^{\prime}, t-t^{\prime}\right) \theta\left(x^{\prime}, t, t^{\prime}\right) \rho\left(x^{\prime}, t^{\prime} \mid x_{0}, t_{0}\right) d t^{\prime}-\right. \\
& \left.\int_{0}^{t} K\left(x, t-t^{\prime}\right) \rho\left(x, t^{\prime} \mid x_{0}, t_{0}\right) \theta\left(x, t, t^{\prime}\right) d t^{\prime}-\omega(x, t) \rho\left(x, t \mid x_{0}, t_{0}\right)\right] d t_{0}+\eta(x, t) .
\end{aligned}
$$

With further simplification, this gives the generalised master equation for an ensemble of particles subject to reactions, forcing and trapping;

$$
\begin{aligned}
\frac{\partial u(x, t)}{\partial t}= & \sum_{x^{\prime}} \lambda\left(x, x^{\prime}, t\right) \int_{0}^{t} K\left(x^{\prime}, t-t^{\prime}\right) \theta\left(x^{\prime}, t, t^{\prime}\right) u\left(x^{\prime}, t^{\prime}\right) d t^{\prime} \\
& -\int_{0}^{t} K\left(x, t-t^{\prime}\right) \theta\left(x, t, t^{\prime}\right) u\left(x, t^{\prime}\right) d t^{\prime}-\omega(x, t) u(x, t)+\eta(x, t) .
\end{aligned}
$$

By taking the special case of no spatial dependence in the memory kernel, i.e. in the waiting times, and taking a jump density that only depends on the differences in location, Eq. (2.13) limits to the Model B generalised master equation (Eq. (26) in [15]). The spatial continuum limit of this special case 
corresponds to the master equation derived in [42]. Also it should be noted that a representation of the master equation, Eq. (2.13), that is closer to the generalized master equation in [43] and [5] can be obtained by identifying

$$
\int_{0}^{t} K\left(t-t^{\prime}\right) \rho\left(x_{i}, t^{\prime}\right) d t^{\prime}=\frac{d}{d t} \int_{0}^{t} M\left(t-t^{\prime}\right) \rho\left(x_{i}, t^{\prime}\right) d t^{\prime} .
$$

The Laplace transform of the memory kernel $M(t)$ is given by

$$
\hat{M}(s)=\frac{\hat{\psi}(s)}{s \hat{\Phi}(s)} .
$$

\section{Fractional Fokker-Planck Equation}

To obtain the corresponding Fokker-Planck equation from the master equation we consider the CTRW on a one dimensional lattice with biased nearest neighbour jumps and the waiting times densities the same at all points. In this case

$$
\lambda\left(x_{i}, x_{i-1}, t\right)=p_{r}\left(x_{i-1}, t\right)
$$

and

$$
\lambda\left(x_{i}, x_{i+1}, t\right)=p_{l}\left(x_{i+1}, t\right)
$$

where $p_{r}(x, t)$ and $p_{l}(x, t)$ denote the probabilities to jump t o the right from $x$ and to the left from $x$ respectively. Eq. (2.13) then reduces to:

$$
\begin{aligned}
\frac{\partial u\left(x_{i}, t\right)}{\partial t}= & \int_{0}^{t} K\left(x_{i-1}, t-t^{\prime}\right) p_{r}\left(x_{i-1}, t\right) \theta\left(x_{i-1}, t, t^{\prime}\right) u\left(x_{i-1}, t^{\prime}\right) d t^{\prime} \\
& +\int_{0}^{t} K\left(x_{i+1}, t-t^{\prime}\right) p_{l}\left(x_{i+1}, t\right) \theta\left(x_{i+1}, t, t^{\prime}\right) u\left(x_{i+1}, t^{\prime}\right) d t^{\prime} \\
& -\int_{0}^{t} K\left(x_{i}, t-t^{\prime}\right) \theta\left(x_{i}, t, t^{\prime}\right) u\left(x_{i}, t^{\prime}\right) d t^{\prime}-\omega\left(x_{i}, t\right) u\left(x_{i}, t\right)+\eta\left(x_{i}, t\right) .
\end{aligned}
$$

The Fokker-Planck equations describing CTRWs with space- and time-dependent forcing can be derived from the generalized master equations by taking a spatial continuum limit. Following [7] we suppose that in the case of an external space- and time-dependent force field,

$$
F(x, t)=-\frac{\partial V(x, t)}{\partial x}
$$

the bias probabilities are given by (near thermodynamic equilibrium) Boltzmann weights

$$
\begin{aligned}
& p_{r}\left(x_{i}, t\right)=\frac{e^{-\beta V\left(x_{i+1}, t\right)}}{e^{-\beta V\left(x_{i+1}, t\right)}+e^{-\beta V\left(x_{i-1}, t\right)}}, \\
& p_{\ell}\left(x_{i}, t\right)=\frac{e^{-\beta V\left(x_{i-1}, t\right)}}{e^{-\beta V\left(x_{i+1}, t\right)}+e^{-\beta V\left(x_{i-1}, t\right)}} .
\end{aligned}
$$

The continuum limit of the generalized master equations is then obtained by writing $x_{i}=x, x_{i \pm 1}=x \pm \Delta x$ and carrying out Taylor series expansions in $x$, retaining leading order terms in $\Delta x$. For a general waiting time distribution, this limit gives us

$$
\begin{aligned}
\frac{\partial u(x, t)}{\partial t}= & \frac{\Delta x^{2}}{2} \frac{\partial^{2}}{\partial x^{2}} \int_{0}^{t} \theta\left(x, t, t^{\prime}\right) u\left(x, t^{\prime}\right) K\left(x, t-t^{\prime}\right) d t^{\prime} \\
& -\beta \Delta x^{2} \frac{\partial}{\partial x}\left(F(x, t) \int_{0}^{t} \theta\left(x, t, t^{\prime}\right) u\left(x, t^{\prime}\right) K\left(x, t-t^{\prime}\right) d t^{\prime}\right) \\
& -\omega(x, t) u(x, t)+\eta(x, t) .
\end{aligned}
$$


We can simplify this further by considering particular cases for the waiting time density. An exponential waiting time density leads to the standard Fokker-Planck equation with additive reactions. A power law waiting time density, such as the Pareto density, at long times leads to the fractional Fokker-Planck equation with reactions. Spatially non-uniform trapping without reactions has been considered in [40].

\subsection{Exponential waiting time density}

The exponential waiting time density is defined by

$$
\psi(x, t)=\gamma(x) e^{-\gamma(x) t} .
$$

The expected value of the waiting time is $\langle\tau(x)\rangle=\frac{1}{\gamma(x)}$ and the variance is $\left\langle\tau(x)^{2}\right\rangle-\langle\tau(x)\rangle^{2}=\frac{1}{\gamma(x)^{2}}$. We also have the Laplace transforms $\hat{\psi}(x, s)=\gamma(x) /(s+\gamma(x)), \hat{\Phi}(x, s)=(1-\psi(x, s)) / s$ and $\hat{K}(x, s)=$ $\hat{\psi}(x, s) / \hat{\Phi}(x, s)=\gamma(x)$. Thus $K(x, t)=\gamma(x) \delta(t)$. Substituting this into Eq. (3.1) yields

$$
\frac{\partial u(x, t)}{\partial t}=\frac{\partial^{2}}{\partial x^{2}}(D(x) u(x, t))-\frac{\partial}{\partial x}\left(\frac{1}{\zeta(x)} F(x, t) u(x, t)\right)-\omega(x, t) u(x, t)+\eta(x, t)
$$

where

$$
D(x)=\lim _{\Delta x,\langle\tau(x)\rangle \rightarrow 0} \frac{\Delta x^{2}}{2\langle\tau(x)\rangle}
$$

and

$$
\zeta(x)=\lim _{\Delta x,\langle\tau(x)\rangle \rightarrow 0} \frac{2\langle\tau(x)\rangle}{\beta \Delta x^{2}}
$$

such that $D(x)$ and $\zeta(x)$ remain finite and differentiable with respect to $x$ [4]. Eq. (3.2) is the standard Fokker-Planck equation with spatially dependent waiting times and the addition of the reactive terms.

\subsection{Power Law waiting time density}

Now we consider a Pareto waiting time density, so that the resulting process will exhibit sub-diffusion. The waiting time density is then given by

$$
\psi(x, t)=\left\{\begin{array}{ll}
\frac{\alpha(x) \tau^{\alpha(x)}}{t^{1+\alpha(x)}} & t \in[\tau, \infty], \\
0 & t \in[0, \tau)
\end{array} \quad \text { for } 0<\alpha(x)<1\right.
$$

with the Laplace transform given by a Tauberian (Abelian) theorem [44]

$$
\hat{\psi}(x, s) \sim 1-\Gamma(1-\alpha(x)) \tau^{\alpha(x)} s^{\alpha(x)}
$$

to leading order in $s$ (large $t)$. We also have $\hat{\Phi}(x, s) \sim \Gamma(1-\alpha(x)) \tau^{\alpha(x)} s^{\alpha(x)-1}$ and $\hat{K}(x, s) \sim$ $\frac{1}{\Gamma(1-\alpha(x))} \frac{1}{\tau^{\alpha(x)}} s^{1-\alpha(x)}$. It follows that

$$
\int_{0}^{t} K\left(x, t-t^{\prime}\right) y\left(x, t^{\prime}\right) d t^{\prime} \approx \frac{1}{\tau^{\alpha(x)} \Gamma(1-\alpha(x))} D_{t}^{1-\alpha(x)} y(x, t)
$$

where $D_{t}^{1-\alpha(x)}$ is the Riemann-Liouville fractional derivative of order $1-\alpha(x)$, assuming that the fractional integral $D_{t}^{-\alpha(x)} y(x, t)$ vanishes at $t=0$. The approximation in Eq. (3.3) becomes exact if the MittagLeffler waiting time density is used in place of the Pareto waiting time density [45]. We rewrite Eq. (3.1) so that the integrals are convolutions with the memory kernel by using Eq. (2.9) to give

$$
\begin{aligned}
\frac{\partial u(x, t)}{\partial t}= & \frac{\Delta x^{2}}{2} \frac{\partial^{2}}{\partial x^{2}} \theta(x, t, 0) \int_{0}^{t} K\left(x, t-t^{\prime}\right) \frac{u\left(x, t^{\prime}\right)}{\theta\left(x, t^{\prime}, 0\right)} d t^{\prime} \\
& -\beta \Delta x^{2} \frac{\partial}{\partial x}\left(F(x, t) \theta(x, t, 0) \int_{0}^{t} K\left(x, t-t^{\prime}\right) \frac{u\left(x, t^{\prime}\right)}{\theta\left(x, t^{\prime}, 0\right)} d t^{\prime}\right)-\omega(x, t) u(x, t)+\eta(x, t) .
\end{aligned}
$$


The fractional Fokker-Planck equation with reactions is then,

$$
\begin{aligned}
\frac{\partial u(x, t)}{\partial t}= & \frac{\partial^{2}}{\partial x^{2}}\left(D_{\alpha(x)} \theta(x, t, 0) D_{t}^{1-\alpha(x)}\left[\frac{u(x, t)}{\theta(x, t, 0)}\right]\right) \\
& -\frac{\partial}{\partial x}\left(\frac{1}{\zeta_{\alpha(x)}} F(x, t) \theta(x, t, 0) D_{t}^{1-\alpha(x)}\left[\frac{u(x, t)}{\theta(x, t, 0)}\right]\right)-\omega(x, t) u(x, t)+\eta(x, t) .
\end{aligned}
$$

where

$$
D_{\alpha(x)}=\lim _{\Delta x^{2}, \tau \rightarrow 0} \frac{\Delta x^{2}}{2 \tau^{\alpha(x)} \Gamma(1-\alpha(x))}
$$

and

$$
\zeta_{\alpha(x)}=\lim _{\Delta x^{2}, \tau \rightarrow 0} \frac{\tau^{\alpha(x)} \Gamma(1-\alpha(x))}{\beta \Delta x^{2}}
$$

such that $D_{\alpha(x)}$ and $\zeta_{\alpha(x)}$ remain finite and differentiable with respect to $x$ [4].

In the limiting case with no reactions, i.e. $\omega(x, t)=0$, and $\eta(x, t)=\delta_{x, x_{0}} \delta\left(t-0^{+}\right)$, Eq. (3.4) can be written,

$$
\frac{\partial u(x, t)}{\partial t}=\frac{\partial^{2}}{\partial x^{2}}\left(D_{\alpha(x)} D_{t}^{1-\alpha(x)} u(x, t)\right)-\frac{\partial}{\partial x}\left(\frac{1}{\zeta_{\alpha(x)}} F(x, t) D_{t}^{1-\alpha(x)} u(x, t)\right) .
$$

This is the fractional Fokker-Planck equation with time- and space-dependent forcing that was first derived, for spatially uniform waiting times, in [7].

\section{Summary}

In this paper we have utilized the continuous time random walk as an underlying stochastic process and we have included reactions as a birth and death process and time- and space-dependent forcing through biased jumps. Using this formalism we have derived the generalised master equation, Eq. (2.13), and the fractional Fokker-Planck equation, Eq. (3.4), for a system of particles subjected to both reactions and external forcing. Under appropriate conditions these equations recover well known special cases; the linear reaction sub-diffusion equation [12], the nonlinear reaction sub-diffusion equation [15], the fractional Fokker-Planck equation with space-dependent forcing [4], and the fractional Fokker-Planck equation with space and time dependent forcing [7]. The effects of reactions and forcing together are not simply additive unless the waiting time density is exponential (see for example Eq. (3.4)). The non-additive coupling of reaction and transport should be carefully considered, in the light of this, in experimental systems where anomalous sub-diffusion is observed.

\section{Appendix: Riemann-Stieltjes Integrals}

We consider the time derivative of Eq. (2.4). Because the integrand is not continuous we cannot simply apply Leibniz rule for differentiating under the integral sign. The discontinuity comes from the flux density at $t=0$. A work around is to first separate out the discontinuity and write

$$
q(x, t)=\delta_{x, x_{0}} \delta\left(t-0^{+}\right)+q^{+}\left(x, t \mid x_{0}, 0\right)
$$

where $q^{+}\left(x, t \mid x_{0}, 0\right)$ is right continuous at $t=0$.

A formal justification of Eq. (2.6) requires the use of Riemann-Stieltjes integrals [46]. It is common in the physics literature to write integrals over probability densities as Riemann integrals as in Eq. (2.4) but it is formally correct to write such integrals as Riemann-Stieltjes integrals. In this case we could write

$$
\rho\left(x, t \mid x_{0}, 0\right)=\int_{0}^{t} \Phi\left(x, t-t^{\prime}\right) \theta\left(x, t, t^{\prime}\right) d Q\left(x, t^{\prime}\right)
$$


where

$$
q\left(x, t \mid x_{0}, 0\right)=\frac{d Q(x, t)}{d t} .
$$

If $\frac{d Q}{d t}$ is bounded and continuous then the Riemann-Stieltjes integral can be written as

$$
\begin{aligned}
\rho\left(x, t \mid x_{0}, 0\right) & =\int_{0}^{t} \Phi\left(x, t-t^{\prime}\right) \theta\left(x, t, t^{\prime}\right) \frac{d Q\left(x, t^{\prime}\right)}{d t^{\prime}} d t^{\prime} \\
& =\int_{0}^{t} \Phi\left(x, t-t^{\prime}\right) \theta\left(x, t, t^{\prime}\right) q\left(x, t^{\prime} \mid x_{0}, 0\right) d t^{\prime}
\end{aligned}
$$

which is an ordinary Riemann integral.

However $\frac{d Q}{d t}$ is not bounded and continuous because of the delta function singularity in the initial condition for $q\left(x, t \mid x_{0}, 0\right)$. We can separate this out in the Riemann-Stieltjes representation be writing

$$
\rho\left(x, t \mid x_{0}, 0\right)=\int_{0}^{t} \Phi\left(x, t-t^{\prime}\right) \theta\left(x, t, t^{\prime}\right) \delta_{x, x_{0}} d H\left(t^{\prime}\right)+\int_{0}^{t} \Phi\left(x, t-t^{\prime}\right) \theta\left(x, t, t^{\prime}\right) d Q^{+}\left(x, t^{\prime}\right)
$$

where the first integral includes the discontinuity from the delta function $\delta\left(t-0^{+}\right)$and the second integral is the continuous part. The function $H(t)$ is the Heaviside function. Note that it is common to write

$$
\int_{0}^{t} \Phi\left(x, t-t^{\prime}\right) \theta\left(x, t, t^{\prime}\right) \delta_{x, x_{0}} d H\left(t^{\prime}\right)=\int_{0}^{t} \Phi\left(x, t-t^{\prime}\right) \theta\left(x, t, t^{\prime}\right) \delta_{x, x_{0}} \delta\left(t^{\prime}-0^{+}\right) d t^{\prime}
$$

since $\delta\left(t-0^{+}\right)=\frac{d H}{d t}$. The left hand side is well defined as a Riemann-Stieltjes integral even though the right hand side is not well defined as a Riemann integral. The justification for this is that the right hand side is always evaluated by the expression on the left hand side. In particular when we evaluate

$$
\int_{0}^{t} \Phi\left(x, t-t^{\prime}\right) \theta\left(x, t, t^{\prime}\right) \delta_{x, x_{0}} \delta\left(t^{\prime}-0^{+}\right) d t^{\prime}=\delta_{x, x_{0}} \Phi(x, t),
$$

we are really evaluating

$$
\int_{0}^{t} \Phi\left(x, t-t^{\prime}\right) \theta\left(x, t, t^{\prime}\right) \delta_{x, x_{0}} d H\left(t^{\prime}\right)=\sum_{t^{\prime}} \Phi\left(x, t-t^{\prime}\right) \theta\left(x, t, t^{\prime}\right) \delta_{x, x_{0}}\left(H\left(t^{\prime}+0\right)-H\left(t^{\prime}-0\right)\right)=\Phi(x, t) \delta_{x, x_{0}}
$$

since $H\left(t^{\prime}+0\right)-H\left(t^{\prime}-0\right)$ is zero everywhere except at $t^{\prime}=0$ when the difference is unity. Therefore it is not possible to evaluate

$$
\int_{0}^{t} \Phi\left(x, t-t^{\prime}\right) \theta\left(x, t, t^{\prime}\right) \delta_{x, x_{0}} \delta\left(t^{\prime}-0^{+}\right) d t^{\prime}
$$

as a Riemann sum. Thus we can write

$$
\rho\left(x, t \mid x_{0}, 0\right)=\Phi(x, t) \delta_{x, x_{0}}+\int_{0}^{t} \Phi\left(x, t-t^{\prime}\right) \theta\left(x, t, t^{\prime}\right) d Q^{+}\left(t^{\prime}\right)
$$

For the continuous part we define $q^{+}\left(x, t \mid x_{0}, 0\right)=\frac{d Q^{+}(x, t)}{d t}$ which is bounded and continuous so that

$$
\rho\left(x, t \mid x_{0}, 0\right)=\Phi(x, t) \delta_{x, x_{0}}+\int_{0}^{t} \Phi\left(x, t-t^{\prime}\right) \theta\left(x, t, t^{\prime}\right) q^{+}\left(x, t^{\prime} \mid x_{0}, 0\right) d t^{\prime}
$$

where the remaining integral is now a standard Riemann integral in agreement with Eq. (2.5) and the result Eq. (2.6) follows as above.

Acknowledgements. This work was supported by the Australian Commonwealth Government (ARC No. DP1094680). The authors are grateful to Trevor Langlands for helpful discussions concerning fractional FokkerPlanck equations. 


\section{References}

[1] A. D. Fokker. Die mittlere energie rotierender elektrischer dipole im strahlungsfeld. Annalen der Physik, vol. 348 , no. 5, (1914), pp. 810-820.

[2] M. Planck, Sitzber. Preu. Akad. Wiss., (1917), p. 324.

[3] H. Risken. The Fokker-Planck equation: Methods of solution and applications. Second Edition., vol. 18. Springer Verlag, 1996.

[4] E. Barkai, R. Metzler, J. Klafter. From continuous time random walks to the fractional Fokker-Planck equation. Phys. Rev. E, vol. 61, no. 1, (2000), p. 132.

[5] I. M. Sokolov, J. Klafter. Field-induced dispersion in subdiffusion. Phys. Rev. Lett., vol. 97, no. 14, (2006), p. 140602.

[6] M. Magdziarz, A. Weron, J. Klafter. Equivalence of the fractional Fokker-Planck and subordinated Langevin equations: The case of a time-dependent force. Phys. Rev. Lett., vol. 101, no. 21, (2008), p. 210601.

[7] B. I. Henry, T. A. M. Langlands, P. Straka. Fractional Fokker-Planck equations for subdiffusion with space- and time-dependent forces. Phys. Rev. Lett., vol. 105, no. 17, (2010), p. 170602.

[8] A. Weron, M. Magdziarz, K. Weron. Modeling of subdiffusion in space-time-dependent force fields beyond the fractional Fokker-Planck equation. Phys. Rev. E, vol. 77, no. 3, (2008), p. 036704.

[9] M. G. Hahn, K. Kobayashi, S. Umarov. Fokker-Planck-Kolmogorov equations associated with time-changed fractional Brownian motion. Proc. Amer. Math. Soc., (2011), pp. 691-705.

[10] V. P. Shkilev. Subdiffusion in a time-dependent force field. J. Exp. Theor. Phys., vol. 114, (2012), p. 830.

[11] B. I. Henry, S. L. Wearne. Fractional reaction-diffusion Physica A, vol. 276, no. 3, (2000), pp. 448-455.

[12] B. I. Henry, T. A. M. Langlands, S. L. Wearne. Anomalous diffusion with linear reaction dynamics: From continuous time random walks to fractional reaction-diffusion equations. Phys. Rev. E, vol. 74, no. 3, (2006), p. 031116.

[13] I. M. Sokolov, M. G. W. Schmidt, F. Sagués. Reaction-subdiffusion equations. Phys. Rev. E, vol. 73, no. 3, (2006), p. 031102 .

[14] T. A. M. Langlands, B. I. Henry, S. L. Wearne. Anomalous subdiffusion with multi-species linear reaction dynamics Phys. Rev. E, vol. 77, no. 2, (2008), p. 021111.

[15] S. Fedotov. Non-Markovian random walks and nonlinear reactions: Subdiffusion and propagating fronts. Phys. Rev. E, vol. 81, no. 1, (2010), p. 011117.

[16] E. Abad, S. B. Yuste, K. Lindenberg. Reaction-subdiffusion and reaction-superdiffusion equations for evanescent particles performing continuous-time random walks. Phys. Rev. E, vol. 81, no. 3, (2010), p. 031115.

[17] G. Bel, E. Barkai. Weak ergodicity breaking in the continuous-time random walk. Phys. Rev. Lett., vol. 94, no. 24, (2005), p. 240602.

[18] M. Magdziarz, A. Weron, K. Burnecki, J. Klafter. Fractional Brownian motion versus the continuous-time random walk: A simple test for subdiffusive dynamics. Phys. Rev. Lett., vol. 103, (2009), p. 180602.

[19] W. Deng, E. Barkai. Ergodic properties of fractional Brownian-Langevin motion. Phys. Rev. E, vol. 79, no. 1, (2009), p. 011112 .

[20] A. Weigel, B. Simon, M. Tamkun, D. Krapf. Ergodic and nonergodic processes coexist in the plasma membrane as observed by single-molecule tracking. Proc. Natl. Acad. Sci., vol. 108, no. 16, (2011), pp. 6438-6443.

[21] T. A. M. Langlands, B. I. Henry. Fractional chemotaxis diffusion equations. Phys. Rev. E, vol. 81, no. 5, (2010), p. 051102 .

[22] S. Fedotov. Subdiffusion, chemotaxis, and anomalous aggregation. Phys. Rev. E, vol. 83, no. 2, (2011), p. 021110.

[23] I. Eliazar, J. Klafter. Anomalous is ubiquitous. Ann. Phys., vol. 326, no. 9, (2011), pp. 2517-2531.

[24] K. Ritchie, X. Y. Shan, J. Kondo, K. Iwasawa, T. Fujiwara, A .Kusumi. Detection of non-Brownian diffusion in the cell membrane in single molecule tracking. Biophysical journal, vol. 88, no. 3, (2005), p. 2266

[30] F. Santamaria, S. Wils, E. De Schutter, G. Augustine. The diffusional properties of dendrites depend on the density of dendritic spines. European Journal of Neuroscience, vol. 34, no. 4, (2011), pp. 561-568.

[26] M. Saxton. Anomalous diffusion due to binding: a monte carlo study. Biophysical journal, vol. 70, no. 3, (1996), pp. 1250-1262.

[27] N. Malchus, M. Weiss. Elucidating anomalous protein diffusion in living cells with fluorescence correlation spectroscopyfacts and pitfalls. J. Fluoresc., vol. 20, (2010), pp. 19-26.

[28] J. H. Jeon, V. Tejedor, S. Burov, E. Barkai, C. Selhuber-Unkel, K. Berg-Sørensen, L. Oddershede, R. Metzler, In vivo anomalous diffusion and weak ergodicity breaking of lipid granules. Phys. Rev. Lett., vol. 106, no. 4, (2011), p. 48103.

[29] F. Santamaria, S. Wils, E. De Schutter, G. J. Augustine. Anomalous diffusion in Purkinje cell dendrites caused by spines. Neuron, vol. 52, no. 4, (2006), pp. 635-648.

[30] F. Santamaria, S. Wils, E. De Schutter, G. J. Augustine. The diffusional properties of dendrites depend on the density of dendritic spines. Eur. J. Neurosci., vol. 34, no. 4, (2011), pp. 561- 568.

[31] B. I. Henry, T. A. M. Langlands, S. L. Wearne. Fractional cable models for spiny neuronal dendrites. Phys. Rev. Lett., vol. 100, no. 12, (2008), p. 128103.

[32] T. A. M. Langlands, B. I. Henry, S. L. Wearne. Fractional cable equation models for anomalous electrodiffusion in nerve cells: infinite domain solutions J. Math. Biol., vol. 59, no. 6, (2009), pp. 761-808.

[33] T. A. M. Langlands, B. I. Henry, S. L. Wearne. Fractional cable equation models for anomalous electrodiffusion in nerve cells: Finite domain solutions. SIAM J. Appl. Math., vol. 71, no. 4, (2011), pp. 1168-1203.

[34] A. Lubelski J. Klafter. Fluorescence recovery after photobleaching: the case of anomalous diffusion. Biophys. J., vol. 94, no. 12 , (2008), pp. 4646-4653. 
[35] A. Kolmogoroff, I. Petrovsky, N. Piscounoff. Étude de l'équation de la diffusion avec croissance de la quantité de matiére et son application á un probléme biologique. Moscow Univ. Bull. Math, vol. 1, (1937), pp. 1-25.

[36] R. Fisher. The wave of advance of advantageous genes. Ann. Hum. Genet., vol. 7, no. 4, (1937), pp. 355-369.

[37] D. Ben-Avraham, S. Havlin. Diffusion and reactions in fractals and disordered systems. Cambridge University Press, 2000 .

[38] M. O. Vlad, J. Ross. Systematic derivation of reaction-diffusion equations with distributed delays and relations to fractional reaction-diffusion equations and hyperbolic transport equations: application to the theory of neolithic transition. Phys. Rev. E, vol. 66, no. 6, (2002), p. 061908.

[39] C. N. Angstmann, I. C. Donnelly, B. I. Henry. Pattern formation on networks with reactions: A continuous time random walk approach. Phys. Rev. E, vol. 87, no. 3, (2012), p. 032804.

[40] S. Fedotov, S. Falconer. Subdiffusive master equation with space-dependent anomalous exponent and structural instability. Phys. Rev. E, vol 85, no. 3, (2012), p. 031132.

[41] H. Scher, M. Lax. Stochastic transport in a disordered solid. I. Theory. Phys. Rev. B, vol. 7, (1973), pp. $4491-4502$.

[42] A. Yadav, W. Horsthemke. Kinetic equations for reaction-subdiffusion systems: Derivation and stability analysis. Phys. Rev. E, vol. 74, no. 6, (2006), p. 066118.

[43] A. V. Chechkin, R. Gorenflo, I. M. Sokolov. Fractional diffusion in inhomogeneous media. J. Phys. A, vol. 38, (2005), p. L679.

[44] B. Berkowitz, A. Cortis, M. Dentz, H. Scher. Modelling non-Fickian transport in geological formations as a continuous time random walk. Rev. Geophys., vol. 44, (2006), p. RG2003.

[45] E. Scalas, R. Gorenflo, F. Mainardi, M. Raberto. Revisiting the derivation of the fractional diffusion equation. Fractals, vol. 11, (2003), pp. 281-289.

[46] T. H. Hildebrandt. Definitions of Stieltjes integrals of the Riemann type. The Amer. Math. Monthly, vol. 45, (1938), p. 265. 